\title{
The Biomechanical Model of the Long Finger Extensor Mechanism and its Parametric Identification
}

\author{
Anton Dogadov, Mazen Alamir, Christine Serviere, Franck Quaine \\ Univ. Grenoble Alpes, GIPSA-Lab, F-38000 Grenoble, France \\ CNRS, GIPSA-Lab, F-38000 Grenoble, France
}

\begin{abstract}
The extensor mechanism of the finger is a structure transmitting the forces from several muscles to the finger joints. Force transmission in the extensor mechanism is usually modeled by equations with constant coefficients which are determined experimentally only for finger extension posture. However, the coefficient values change with finger flexion because of the extensor mechanism deformation. This induces inaccurate results for any other finger postures. We proposed a biomechanical model of the extensor mechanism represented as elastic strings. The model includes the main tendons and ligaments. The parametric identification of the model in extension posture was performed to match the distribution of the forces among the tendons to experimental data. The parametrized model was used to simulate three degrees of flexion. Furthermore, the ability of the model to reproduce how the force distribution in simulated extensor mechanism changes according to the muscle forces was also demonstrated. The proposed model could be used to simulate the extensor mechanism for any physiological finger posture for which the coefficients involved in the equations are unknown.
\end{abstract}

Keywords - biomechanical modelling, finger modelling, extensor mechanism, tendons 


\section{INTRODUCTION}

The extensor mechanism (EM) of the finger (the extensor apparatus, extensor expansion, extensor assembly, dorsal aponeurosis, etc.) is a complex anatomical structure which transmits the forces of several extrinsic and intrinsic hand muscles to the finger joints (See Fig. 1a,b in the Method section). It is situated on the dorsal surface of the finger bones and is involved in both extension and flexion of the finger joints.

Owing to its important role in force transmission, the EM has been incorporated into biomechanical models of the finger (Sancho-Bru et al., 2001; Vigouroux et al., 2007; Hu et al., 2014). For the extended posture it is usually modeled by an equation system proposed by Chao et al. (1989) which represents the internal-force distribution in the tendon network (see eq. 3). To take into account the EM for other postures it is usually represented as a 3D-network of extensible or non-extensible strings placed on finger bones at given joint angles. Some models of this type may include only tendons as the individual elements (Giurintano and Sancho, 1999; Valero-Cuevas and Lipson, 2004; Hu et al., 2014). Some models also take ligaments into account as separated elements or incorporate them into a model as constraints (Leijnse and Spoor, 2012; Sachdeva et al., 2015; Vaz et al., 2015). The models may contain a high number of elements, which allows modeling of the EM with a high degree of precision in order to simulate the clinical deformities of the mechanism (Sachdeva et al., 2015). However, such 3D-models contain many unknown parameters that should be determined. It seems that most studies in this field have only focused on EM modelling but not on its parametrization and validation.

The purpose of this study was to create a numeric model of the long finger EM that includes main tendons and ligaments as well as to perform a parametric identification of the model to match the force distribution given by Chao equation system for the extension posture (4). The proposed model of the EM with the parametrization algorithm aims to contribute to improving the relevance of biomechanical models of finger in simulating all feasible posture given in the literature for normal finger (Leijnse et al., 2010). 
Model

The EM was modeled as a network of elastic bands. Fig. 1c shows the components of the EM included in the model. Each EM component, or band, was discretized by the chain of the points, connected by elastic elements. Hence, the EM position was represented by the array $\mathbf{x}$ of $\mathrm{x}, \mathrm{y}, \mathrm{z}$-coordinates of points forming the EM. Each two sequential points, discretizing an EM band, were connected by a spring with a linear elasticity model. The bones were modeled by cylinders and spheres and completed by three auxiliary cylinders, perpendicular to the bones ( $a$, $b, c$ in Fig. 1c), which replace the function of pulleys or condyles.

The forces of dorsal ulnar interosseous $u i$, extensor digitorum $e d$, dorsal radial interosseous $r i$, and lumbrical $l u$ muscle were used as the external ones. The fraction of these

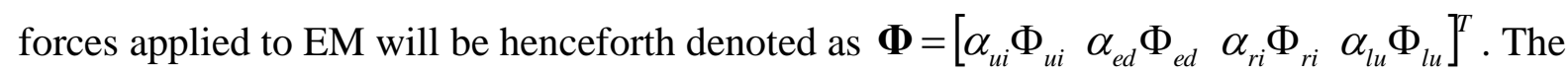
muscle forces $\Phi_{j}$ ranged from 2.45-7.35 N and were taken from the cadaveric studies by GarciaElias et al. (1991) and by Hurlbut et al. (1995). The weight coefficients $\boldsymbol{\alpha}=\left[\begin{array}{llll}0.626 & 0.500 & 0.267 & 1.00\end{array}\right]^{T}$ were used to take into account the fact that there is a fraction of $u i, e d$ and $r i$-muscle force that is applied to the base of metacarpal bone and is not transmitted by the tendons of the EM (Eyler and Markee, 1954; Zancolli, 1979). Their values were estimated from Chao et al. (1989).

The principle of Minimum Potential Energy was used to find the equilibrium state, in which the EM internal forces balance the muscle forces. At iteration $k+1$ the potential energy $(P E)$ of the EM was calculated as a sum of the strain energy $(S E)$ of the spring system and work potential of the muscle forces $(W P)$ :

$$
P E\left(\mathbf{x}_{k+1}\right)=S E\left(\mathbf{x}_{k+1}\right)+W P\left(\mathbf{x}_{k+1}, \mathbf{x}_{k}, \mathbf{\Phi}\right)+g\left(\mathbf{x}_{k+1}\right),
$$

where $g$ represents penalty term, which was added to enforce the constraint expressing the fact that the points forming EM should not penetrate the bone surface. 
51 The Broyden-Fletcher-Goldfarb-Shanno method (BFGS) was used to find the minimum of

52 potential energy. The array $\mathbf{x}$ at iteration $k+1$ was calculated as:

$$
\mathbf{x}_{k+1}=\mathbf{x}_{k}-\mathbf{H}_{k} \cdot \nabla P E,
$$

53

$$
\left\{\begin{array}{l}
F_{t e}=1.000 F_{u b}+1.000 F_{r b} \\
F_{r b}=0.167 \Phi_{e d}+0.133 \Phi_{r i}+0.667 \Phi_{l u} \\
F_{u b}=0.313 \Phi_{u i}+0.167 \Phi_{e d} \\
F_{m e}=0.313 \Phi_{u i}+0.167 \Phi_{e d}+0.133 \Phi_{r i}+0.333 \Phi_{l u}
\end{array}\right.
$$
$C_{1} \ldots C_{11}$ will be further denoted as a vector $\mathbf{C}=\left[C_{1} \ldots C_{11}\right]^{T}$, which determine the force 1989):

where $F_{i}$ are the internal forces in the tendons, $\Phi_{i}$ are the muscle forces applied to the EM. The internal force subscripts are abbreviations of tendon names, listed in Table 1 . The coefficients distribution among the bands of the EM. The values of $\mathbf{C}$ depend on the properties of the tendons and the angles between them. These values were measured for the extended posture (Chao et al.

63 The experimentally defined coefficients in (4) will be denoted as a vector $\hat{\mathbf{C}}=[1.00 \ldots 0.33]^{T}$. For each simulation, the coefficients, describing the force distribution in simulated EM

65 were estimated and denoted as $\mathbf{C}^{*}=\left[C_{1}^{*} \ldots C_{11}^{*}\right]^{T}$. There are more unknown coefficients than equations in (3). Hence, to determine $\mathbf{C}^{*}$ the EM should be simulated for different muscle force 
67 sets, which increases the number of equations in (3). The forces from the right part of (3) after equation number increase were denoted as the matrix $\mathbf{B}^{*}$, and the forces from the left part were denoted as the matrix-column $\mathbf{A}^{*}$. As $\mathbf{A}^{*}$ was calculated to be accurate within a certain degree of error, the values of the coefficients were estimated by Tikhonov regularization and were denoted $\mathbf{C}^{*}:$

$$
\mathbf{C}^{*}=\underset{\mathbf{C}}{\arg \min }\left(\left\|\mathbf{B}^{*} \mathbf{C}-\mathbf{A}^{*}\right\|^{2}+\alpha\|\mathbf{C}\|^{2}\right)
$$

where a regularization parameter $\alpha$ was set to 0.01 .

It should be noted, that the muscle force sets, applied to the model to estimate $\mathbf{C}^{*}$, should be relatively close, otherwise it can modify the EM configuration and bias $\mathbf{C}^{*}$. The study of the muscle force value effect on $\mathbf{C}^{*}$ is described in the next subsection.

A model parametrization was performed to make the model-describing coefficients $\mathbf{C}^{*}$ fit the experimentally measured coefficients $\hat{\mathbf{C}}$. The lengths of two intercrossing bands, extensor lateral band $e l$ and interosseous medial band $i m$ (2 and 5 in Fig. 1) were chosen as the identified parameters, denoted by a vector $\mathbf{I}=\left[\begin{array}{ll}l_{e l} & l_{i m}\end{array}\right]^{T}$. These tendons were chosen, because they have a strong influence on the EM configuration (Schultz et al., 1981). The root-mean-square error between $\mathbf{C}^{*}$ and $\hat{\mathbf{C}}$ was used as a cost-function $J(\mathbf{l})$,

$$
J(\mathbf{l})=\sqrt{\frac{\sum_{q=1}^{Q}\left(\hat{C}_{q}(\mathbf{l})-C_{q}^{*}(\mathbf{l})\right)^{2}}{Q}}
$$

where $Q$ denotes the number of coefficients $(Q=11)$.

The Nelder-Mead algorithm (fminsearch function in Matlab R2012b, MathWorks, Natwick, MA) was used to solve the unconstrained problem of $J(\mathbf{l})$ minimization.

$$
\mathbf{l}_{\text {opt }}=\underset{\mathbf{l}}{\arg \min } J(\mathbf{l}),
$$


The starting point of the algorithm corresponded to such EM configuration, in which all lateral and medial bands were tight (2 and 3, 4 and 5 in Fig. 1).

\section{Sensitivity analysis}

After the model was parametrized, the sensitivity analysis was performed for the fully extended posture. As the EM is deformable, the force distribution among the tendons could vary when the muscle forces change. For the EM model this distribution is represented by $\mathbf{C}^{*}$. The sensitivity of $\mathbf{C}^{*}$ to the variation in muscle force values, which are the model inputs, was calculated as:

$$
S I\left(C_{q}^{*}\right)_{j}=\frac{C_{q}^{*}\left(\Phi_{1}, \ldots, \Phi_{j}+\Delta \Phi_{j}, \ldots, \Phi_{n}\right)-C_{q}^{*}\left(\Phi_{1}, \ldots, \Phi_{j}-\Delta \Phi_{j}, \ldots, \Phi_{n}\right)}{2 \Delta \Phi_{j}}
$$

where $\Delta \Phi_{j}$ was $0.5 \mathrm{~N}$. The positive value of a sensitivity index $S I\left(C_{q}^{*}\right)_{l}$ indicates that $q$-th coefficient increases when the force of $j$-th muscle increases.

\section{RESULTS}

The results address first the parametrization and the simulation of different postures using the parametrized model and, finally, the sensitivity analysis. Moreover, we used the parametrized model to estimate the coefficients $\mathbf{C}^{*}$ for a sequence of physiological postures (Harris and Rutledge, 1972). These coefficients are presented as a supplementary material for this paper. Their values need to be interpreted with caution as they strongly depend on MCP, PIP, and DIP joint angles (Wook et al., 2008), and there may be a lot of feasible combinations of these angles (Leijnse et al., 2010). Hence, given coefficients can be used for the investigated postures; otherwise the coefficients should be recalculated accordingly to the proposed method.

\section{Parametrization}

The identified tendon lengths for $l_{e l}$ and $l_{i m}$ are given in Table 2 . The identified value of $l_{i m}$ is $10 \%$ lower than the value defined by anatomical survey (Table 1$)$. No comparison was possible for $l_{e l}$ as no published data exists. The identified parameters results in a very good fit for 
$C_{1}^{*}, C_{2}^{*}, C_{6}^{*}$, and $C_{8}^{*}$ (Fig. 2). Coefficients $C_{1}^{*}$, and $C_{2}^{*}$ represent the fraction of the force in

109 ulnar and radial lateral band $r b$ and $u b$ (9 in Fig. 1) transmitted to terminal extensor tendon te (10 in Fig. 1). $C_{6}^{*}$ and $C_{8}^{*}$ represent the fraction of the $u i$-muscle transmitted to $u b$ and medial extensor tendon me (6 in Fig.1). The major difference concerns coefficients $C_{5}^{*}$, and $C_{11}^{*}$, related to the fraction of $l u$-muscle transmitted to $r b$ and $m e$-tendon. The identified parameter set was used to model three degrees of extension observed in (Garcia-Elias et al., 1991; Hurlbut and Adams, 1995), which are full flexion, mid-flexion and full extension. The simulation results are shown in Fig. 3. The changes of the EM configuration with extension can be seen from the figure Fig. 3. The extensor hood shifts proximally ( 1 in Fig. 3b) while $u b$ and $r b$-tendon shift medially (9 in Fig. 3b). The retention apparatus is also affected by changes: the retinacular ligament ( 7 in Fig. $3 d$, e) becomes tight and triangular ligament (8 in Fig. 3d, e) relaxes with extension.

\section{Sensitivity analysis}

Fig. 4 represents sensitivity indices $S I\left(C_{q}^{*}\right)_{j}$, which show how the values of $\mathbf{C}^{*}$ change with respect to muscle force variation. It can be seen that the most significant changes are

In this study, a biomechanical model of the EM was proposed to simulate the changes in the EM configuration with posture in order to better understand force transmission in the bands strings to simulate the change in configuration according to different postures. One improvement and thus accurately model the finger biomechanics behavior. The model was inspired by the model proposed by Valero-Cuevas and Lipson (2004). The EM was represented as a network of lies in the inclusion of the triangular and oblique retinacular ligaments in the model. 

among the tendons to experimental data for the fully extended posture. Finally, we calculated the sensitivity of the parametrized model to the variation of the muscle force values.

\section{Parametrization}

The parametric identification of the model was performed to make the coefficients

discrepancies between the simulations and the measurements concern the $C_{5}^{*}$ and $C_{11}^{*}$ coefficients, associated with the force, transmitted from $l u$-muscle. One possible explanation of these differences may be an imperfection of $l u$-muscle representation, which is the small muscle with a wide range of origin variation (Goldberg, 1970).

\section{Sensitivity analysis}

We studied how much the model-describing coefficients are sensitive to muscle force

values. It appears that great differences can be observed for $C_{3}^{*}, C_{4}^{*}$, and $C_{5}^{*}$. As a conclusion, the coefficients given by Chao et al. (1989) and used in most finger models could be inexact in some range of muscle forces. These results show how the force sharing among the EM bands changes if the force sharing among the muscles also changes. These findings could be important for accurate finger biomechanical modeling. This study should be distinguished from that of previous authors (Hurlbut and Adams, 1995; Wook et al., 2008) that showed that the force distribution among the EM tendons does not depend on the overall muscle force level when the force distribution among the muscles remained constant.

\section{Perspectives}

The proposed model could be a tool to simulate the EM deformation during finger flexion-extension and change in force distribution. It may be directly incorporated into finger model or used to recalculate the coefficients for any physiological finger posture, muscle force level, and bone geometry. Moreover, it could improve the precision of the existing 
by optimization for required posture (Sancho-Bru et al., 2001; Vigouroux et al., 2007).

Furthermore, more complex models, representing the EM as a membrane instead of the set of the elastic bands may be created. Moreover, the current model uses several assumptions, which can be subsequently removed as limiting the model accuracy. These assumptions concern:

1. Bones. The friction between the EM and the surface was not taken into account. The bones were modeled as cylinders with spheres at the ends. However, the joint surface of finger bones is irregular, which results in increase of the digit skeleton length with flexion. Zancolli (1979) reported a 20mm difference in finger skeleton length between full flexion and full extension postures. To minimize the influence of this effect the radius of the spheres were chosen as the mean radius of joint surfaces, which reduces the mean error among all postures.

2. Muscles. The muscle forces were represented by the vectors directed to the center of the muscle body. In the case of the extrinsic ed-muscle, the force vector was directed along the long extensor tendon. To increase the model precision the muscle body shift during the flexion should be taken into account. This is particularly true for lumbrical muscles, which shift distally during the finger flexion movement and change the orientation of the force.

3. Tendons. The tendons were modeled as springs with a linear elasticity model. This assumption conforms to experimental data. Garcia-Elias et al (1991) showed that the extensor mechanism tendons demonstrate a behavior, close to a liner elasticity model, in a physiological force range.

\section{CONFLICT OF INTEREST}

The authors of this manuscript declare that there are no conflicts of interest

\section{ACKNOWLEDGMENTS}

The authors thank Prof. François Moutet (CHU Grenoble), Dr. Lionel Reveret (INRIA

Rhône-Alpes), and Dr. Isabelle Sivignon (GIPSA-Lab) for their valuable comments. 
hand. A basic research study. World Scientific, Singapore. the fingers. J. Bone Jt. Surg. - Am. Vol. 36, 1-18. Garcia-Elias, M., An, K.N., Berglund, L., Linscheid, R.L., Cooney, W.P., Chao, E.Y.S,

1991. Extensor mechanism of the fingers. I. A quantitative geometric study. J. Hand Surg. Am. $16,1130-1136$.

Garcia-Elias, M., An, K.-N., Berglund, L.J., Linscheid, R.L., Cooney, W.P., Chao,

E.Y.S., 1991. Extensor mechanism of the fingers. II. Tensile properties of components. J. Hand Surg. Am. 16, 1136-1140.

finger's interphalangeal joints coupling mechanism — variability, flexion - extension 

morphology from measured coupled interphalangeal joint angle trajectories - a generic 2D kinematic model. J. Biomech. 45, 569-578. properties vary for different regions of the finger extensor apparatus. J. Biomech. 47, 30943099.

Sachdeva, P., Sueda, S., Bradley, S., Fain, M., Pai, D.K., 2015. Biomechanical simulation and control of hands and tendinous systems. ACM Trans. Graph. 34. 
235 Company, Philadelphia; Toronto. 
Fig. $1 \mathrm{a}-$ the simplified anatomic view of the extensor mechanism of the left hand long finger (dorsal view). 1b - lateral view. The muscles (blue), tendons (green), and ligaments (light blue) are shown. 1c - the schematic view of the proposed model. The structures included in the model are denoted by numbers from 1 to 10 and listed in Table 1. For pair bands, located at both ulnar and radial side, only radial bands are enumerated.

Fig. 2. The experimentally defined coefficients $\hat{C}_{q}$ from the equations (4) shown in comparison with the corresponding model-characterizing coefficients $C_{q}^{*}$. The former values are depicted by filed bars, the latter values are depicted by the hatched bars.

Fig. 3. The extensor mechanism simulation results during three finger postures. Left to right:(a) full flexion $\left[\mathrm{DIP}=90^{\circ} ; \mathrm{PIP}=90^{\circ} ; \mathrm{MCP}=90^{\circ}\right.$, (b) mid-flexion $\left[\mathrm{DIP}=30^{\circ} ; \mathrm{PIP}=45^{\circ}\right.$; $\left.\mathrm{MCP}=45^{\circ}\right]$, (c) full extension $\left[\mathrm{DIP}=0^{\circ} ; \mathrm{PIP}=0^{\circ} ; \mathrm{MCP}=0^{\circ}\right]$; the scaled-up retinacular and triangular ligaments during full flexion (d) and full extension (e).

Fig. 4. The sensitivity index SI, showing the sensitivity of model-characterizing coefficients $C_{q}^{*}$ to variation of muscle force values. 
a)

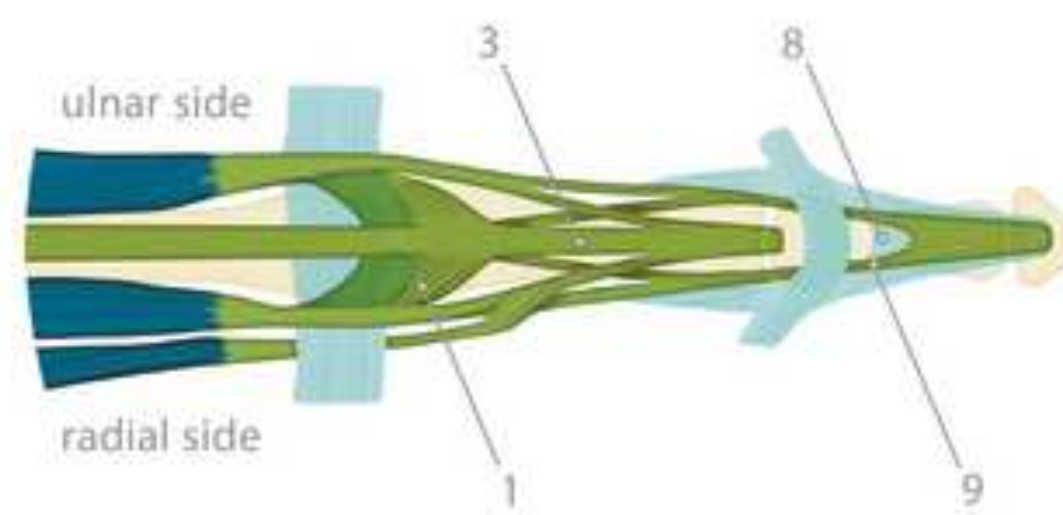

b)

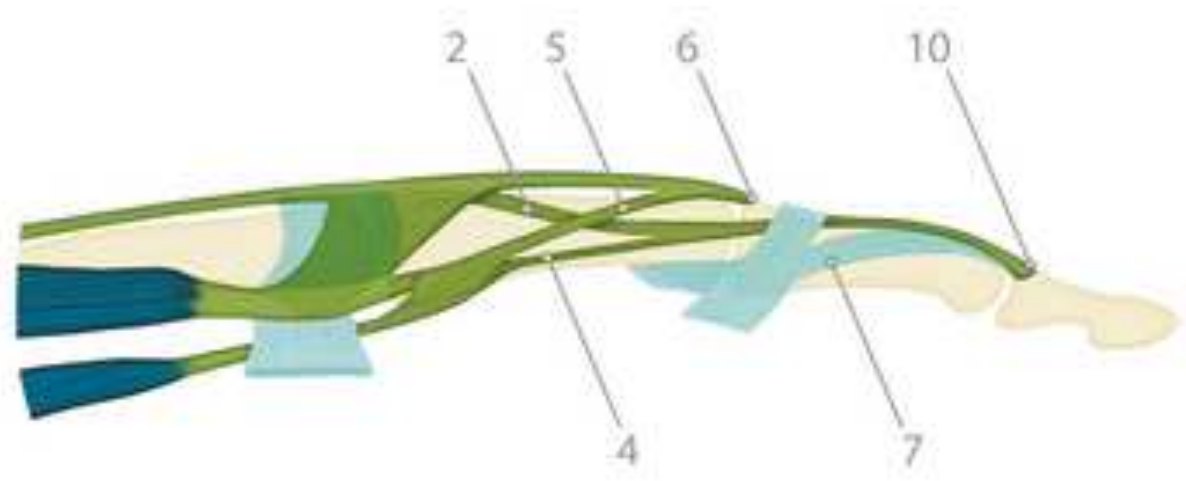

C)

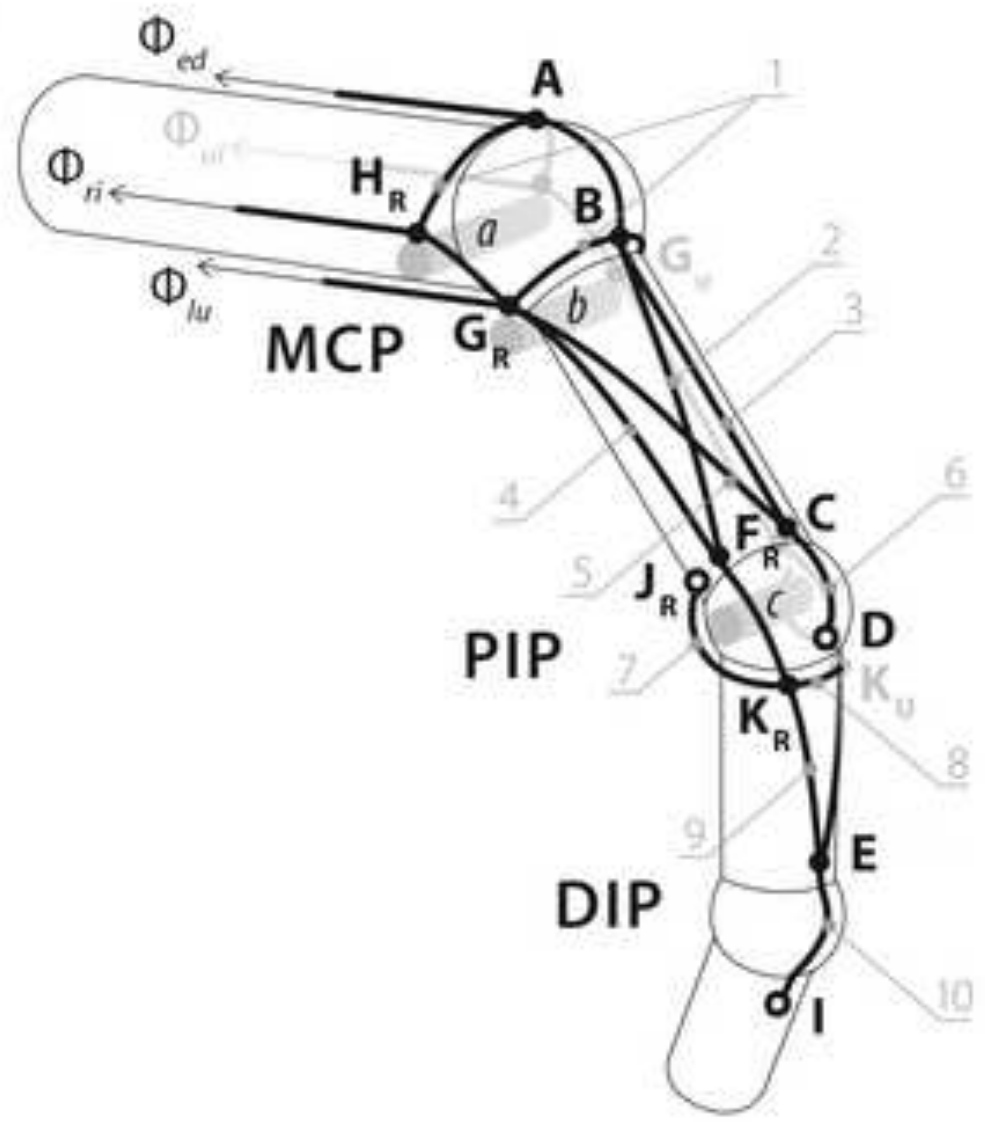


Figure 2

Click here to download high resolution image

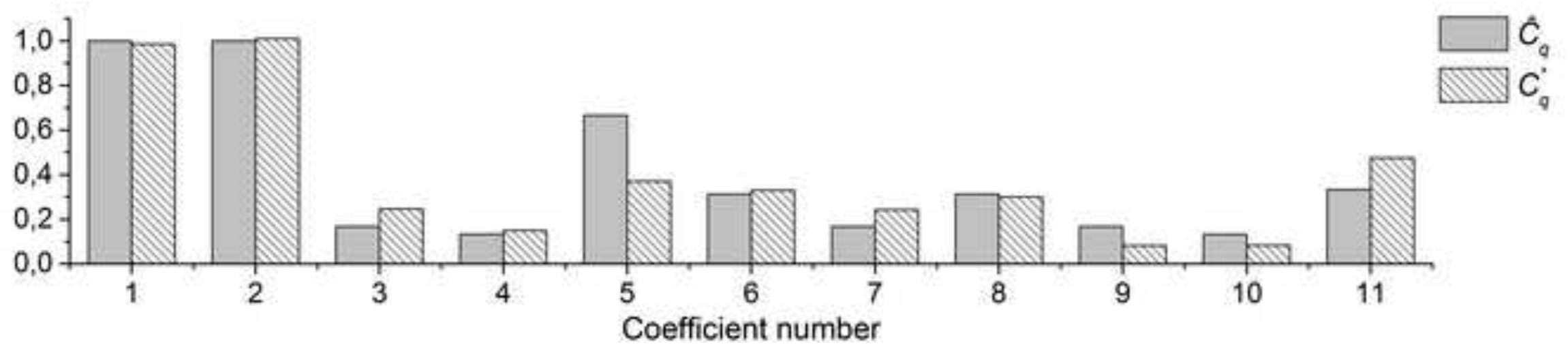


a)

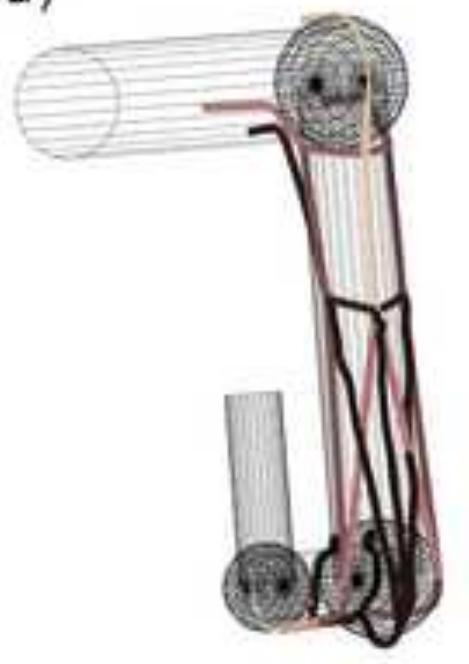

b)

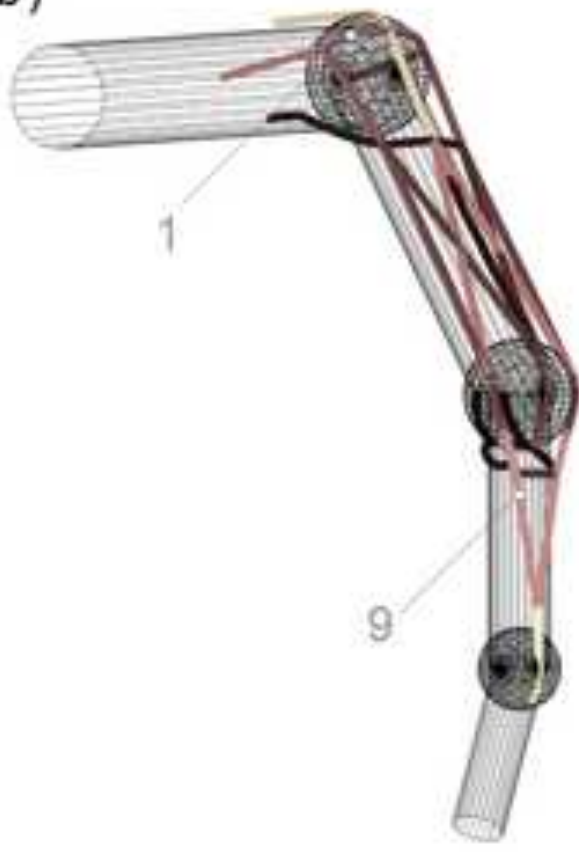

c)

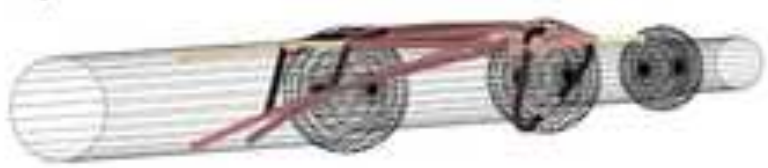

d)

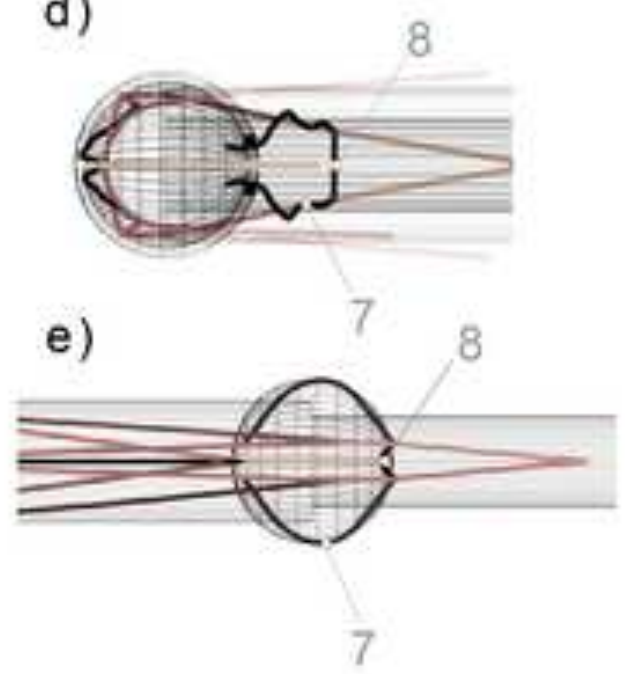

$-8|N|$ 
Click here to download high resolution image

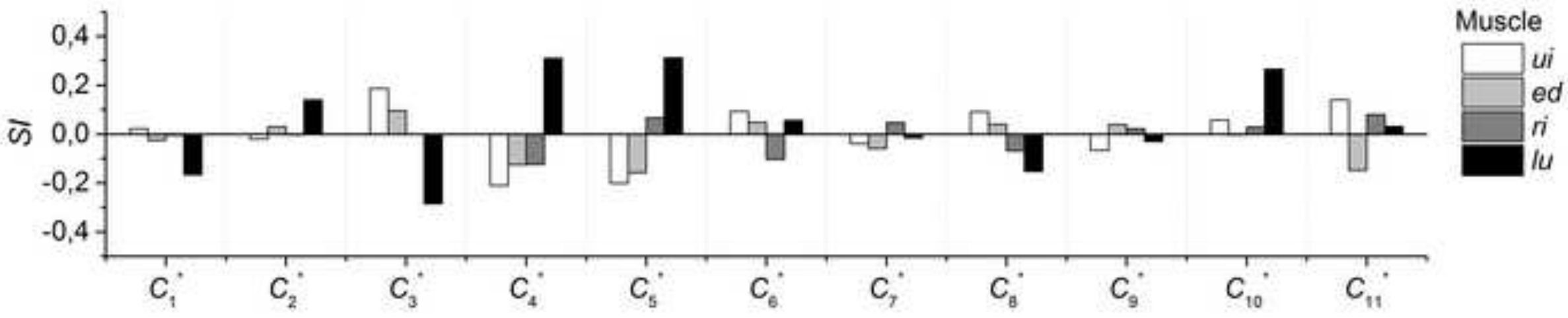


Table 1. The elements of the extensor mechanism, included into the model

\begin{tabular}{|c|c|c|c|c|c|}
\hline $\begin{array}{l}\text { Element } \\
\text { number }\end{array}$ & Extensor mechanism element name & Abbreviation & $\begin{array}{l}\text { Length } \\
\text { (mm) }\end{array}$ & $\begin{array}{c}\text { Thickness } \\
\text { (mm) }\end{array}$ & $\begin{array}{l}\text { Modulus of } \\
\text { elasticity (MPa) }\end{array}$ \\
\hline \multirow{2}{*}{1} & $\begin{array}{l}\text { Proximal interosseous hood, radial } \\
\text { and ulnar }\end{array}$ & - & $18.4 \pm 2.5^{\mathrm{a}}$ & \multirow{2}{*}{$1.19 \pm 0.33^{\mathrm{c}}$} & \multirow{2}{*}{$64.87 \pm 29.30^{c}$} \\
\hline & $\begin{array}{l}\text { Distal interosseous hood, radial and } \\
\text { ulnar }\end{array}$ & - & $18.5 \pm 3.0^{\mathrm{a}}$ & & \\
\hline 2 & Extensor lateral band, radial and ulnar & el & N/A & N/A & N/A \\
\hline 3 & Extensor medial band & - & $33.6 \pm 4.4^{\mathrm{a}}$ & $1.38 \pm 0.29^{\mathrm{c}}$ & $114.03 \pm 61.34^{\mathrm{c}}$ \\
\hline 4 & $\begin{array}{l}\text { Interosseous lateral band, radial and } \\
\text { ulnar }\end{array}$ & - & $37.1 \pm 2.6^{\mathrm{a}}$ & N/A & N/A \\
\hline 5 & $\begin{array}{l}\text { Interosseous medial band, radial and } \\
\text { ulnar }\end{array}$ & im & $36.2 \pm 1.9^{\mathrm{a}}$ & N/A & N/A \\
\hline 6 & Medial extensor tendon & $m e$ & $11.2 \pm 1.8^{\mathrm{a}}$ & $1.20 \pm 0.31^{\mathrm{c}}$ & $125.31 \pm 62.06^{\mathrm{c}}$ \\
\hline 7 & $\begin{array}{l}\text { Oblique retinacular ligament, radial } \\
\text { and ulnar }\end{array}$ & - & $15^{\mathrm{d}}$ & N/A & N/A \\
\hline 8 & Triangular ligament & - & $5.4 \pm 1.1^{\mathrm{b}}$ & N/A & N/A \\
\hline 9 & Lateral band, radial and ulnar & $r b$ and $u b$ & $18.4 \pm 4.3^{\mathrm{a}}$ & $1.20 \pm 0.39^{\mathrm{c}}$ & $157.02 \pm 138.37^{\mathrm{c}}$ \\
\hline 10 & Terminal extensor tendon & te & $10.1 \pm 2.6^{b}$ & $1.07 \pm 0.20^{\mathrm{c}}$ & $96.97 \pm 51.29^{\mathrm{c}}$ \\
\hline
\end{tabular}

a(Garcia-Elias et al., 1991);

b(Schweitzer and Rayan, 2004);

'(Qian et al., 2014);

d(Shrewsbury and Johnson, 1977);

N/A denotes non-available data 
Table 1. The parametrization results

\begin{tabular}{|l|l|}
\hline Length of extensor lateral band $l_{e l}(\mathrm{~mm})$ & 38.0 \\
\hline Length of interosseous medial band $l_{i m}(\mathrm{~mm})$ & 32.7 \\
\hline Cost-function $J$ & 0.11 \\
\hline
\end{tabular}


Supplementary Material
Click here to download Supplementary Material: Table 3.docx

Click here to download Supplementary Material: Table 3.docx

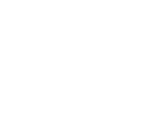

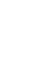

$\sqrt{2}$

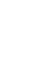

(1)

(1)

(1)

(1)

(1)

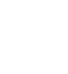

.

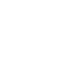

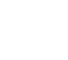

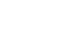

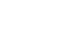

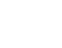

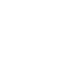

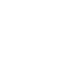

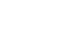

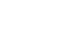

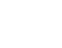

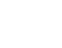

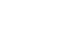

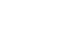

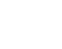

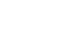

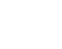

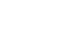

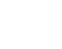

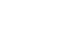

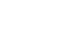

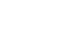

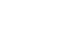

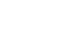

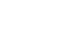

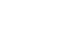

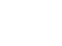

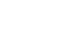

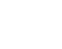

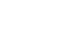

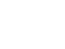

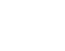

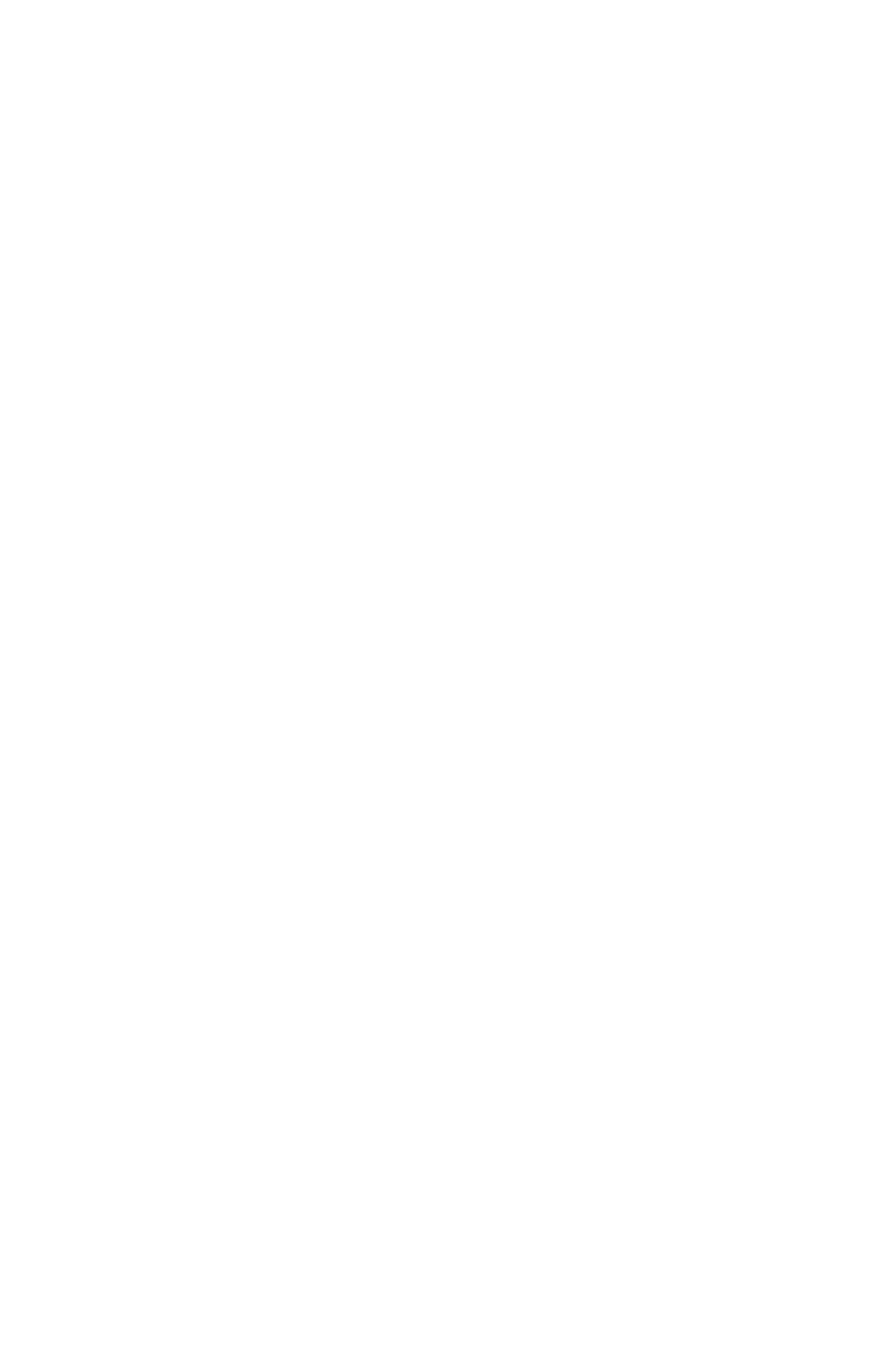

